\title{
Systemic lupus erythematosus, pulmonary hypertension, and left recurrent laryngeal nerve palsy
}

\author{
O M ASZKENASY, T J CLARKE, P HICKLING, AND A J MARSHALL \\ From the Departments of Rheumatology and Cardiology, Plymouth General Hospital
}

SUMMARY Pulmonary hypertension in systemic lupus erythematosus (SLE) is rare in the $\dot{i}_{i}^{\dot{\omega}}$ absence of parenchymal lung disease. We report such a case, which was complicated by the $\stackrel{f}{\circ}$ subsequent development of a left recurrent laryngeal nerve palsy, and in which there was a strong $ᄋ$ family history of SLE.

Key words: pulmonary artery.

\section{Case report}

A 41 year old woman presented in 1979 with polyarthritis, alopecia, and a photosensitive rash. She had a longstanding history of Raynaud's phenomenon and menorrhagia. Two of her sisters suffered from SLE, one of them having died from renal complications of the disease. Her daughter also suffers from Raynaud's phenomenon, arthralgia, and has a positive antinuclear antibody test. Investigations showed haemoglobin $(\mathrm{Hb}) 9.7 \mathrm{~g} / \mathrm{dl}$ $(97 \mathrm{~g} / \mathrm{l})$ and iron deficiency, leucocytes $4 \cdot 3 \times 10^{9} / 1$ with the absolute lymphocyte count being reduced at $0.9 \times 10^{9} / 1$, neutrophils $3.1 \times 10^{9} / 1$, eosinophils $0.35 \times 10^{9} / 1$, basophils $0.05 \times 10^{9} / 1$. Platelets were moderately reduced at $136 \times 10^{9} / 1$, erythrocyte sedimentation rate was $78 \mathrm{~mm} / \mathrm{h}$, antinuclear antibody titre $1 / 640$ (substrate rat liver, staining pattern homogeneous), anti-double-stranded DNA antibody titre $1 / 160$ (Farr assay). Chest radiograph was normal.

A diagnosis of SLE was made and treatment was started with prednisolone, hydroxychloroquine, and iron supplements. In 1981 she developed angina of effort. The electrocardiogram showed right ventricular hypertrophy and the chest radiograph showed an increased cardiac diameter. She was treated with nifedipine initially, but could not

Accepted for publication 11 August 1986

Correspondence to Dr P Hickling, Mount Gould Hospital, Mount Gould Road, Plymouth PL4 7QD. tolerate this because of headaches, and isosorbide dinitrate was given.

In November 1985 she was admitted to hospital with a three week history of increasing dyspnoea, ankle oedema, and worsening angina. On admission $\mathbb{D}$ she looked unwell with pallor, dyspnoea, and evidence of right ventricular failure. Investigations showed $\mathrm{Hb} 7.8 \mathrm{~g} / \mathrm{dl}(78 \mathrm{~g} / \mathrm{l})$, leucocytes $3.8 \times 10^{9} / 1=$ (differential count: lymphocytes $0 \cdot 8 \times 10^{9} / 1$, neutrophils $2.7 \times 10^{9} / 1$, eosinophils $0.2 \times 10^{9} / 1$, basophils $\left.0 \cdot 1 \times 10^{9} / 1\right)$, platelets $141 \times 10^{9} / 1$. Chest radiograph showed cardiomegaly, widening of the anterior mediastinum, and clear lung fields. Electrocardio- $\underline{3}$ graphy showed increased right ventricular hyper- $\delta$ trophy, which was confirmed on echocardiography. A ventilation and perfusion lung scan was normal.

Tests for the lupus anticoagulant and cardiolipin? antibodies (Venereal Disease Research Laboratoryo test for syphilis) were negative, though these tests were performed shortly after treatment was started. O Tissue type was HLA-A11, A28, B44(12), B35. N

She was given prednisolone $60 \mathrm{mg} /$ day, N frusemide, and transfused with four units of blood. There was a marked clinical improvement initially, but three days later she developed hoarseness. Indirect laryngoscopy showed complete paralysis of the left vocal cord. Thoracic computed tomography showed greatly dilated proximal pulmonary arteries. $\overline{0}$ Right heart catheterisation was performed and confirmed the presence of severe pulmonary $\frac{\Omega}{\mathbb{D}}$ hypertension. The pulmonary arterial pressure was $\varrho$ $135 / 58 \mathrm{mmHg}$ and the right ventricular pressure 


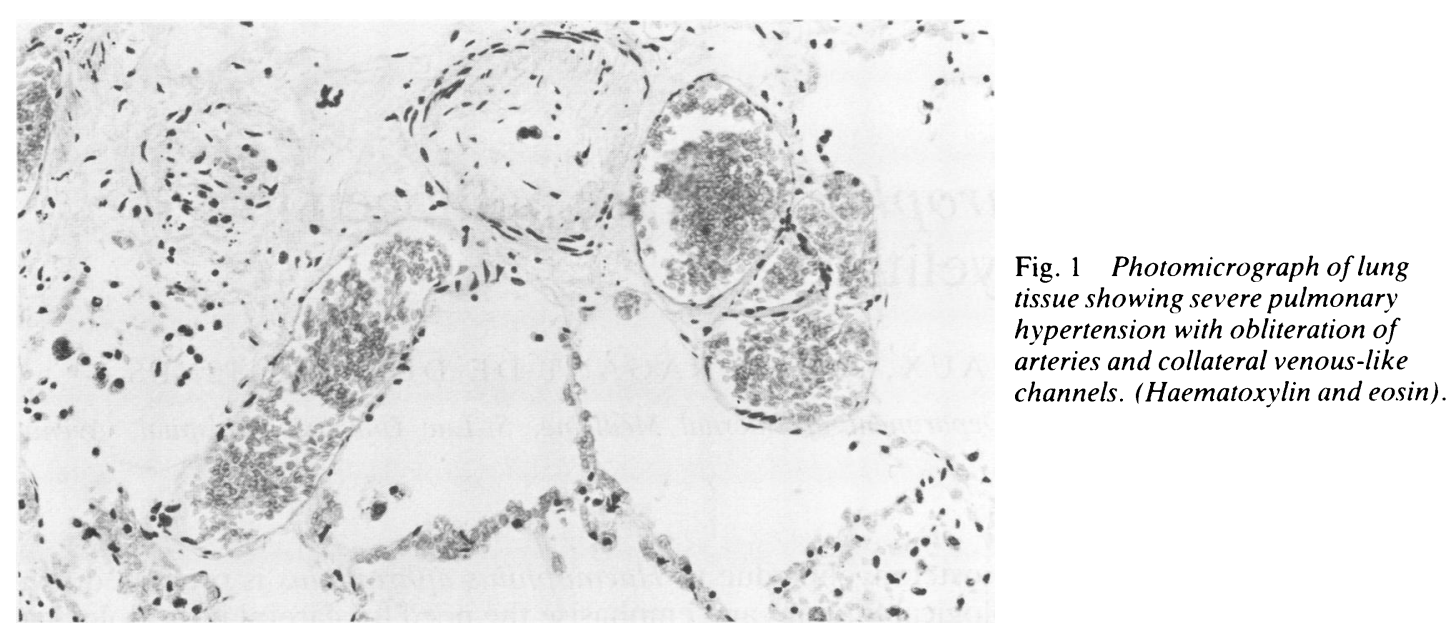

$128 / 1 \mathrm{mmHg}$. The mean pulmonary wedge pressure was normal at $4 \mathrm{mmHg}$. Pulmonary function tests showed a mild restrictive defect with a normal gas transfer.

Nifedipine was added to her regimen and was tolerated on this occasion. Her voice gradually improved. After a brief discharge from hospital she was readmitted with severe right heart failure and died.

A postmortem examination showed that the left recurrent laryngeal nerve was compressed against the ligamentum arteriosum by the dilated left pulmonary artery. The nerve showed pronounced myelinolysis with dystrophic calcification in the adjacent adipose tissue. The lung parenchyma was normal. There were widespread changes of severe pulmonary hypertension with obliteration of small muscular arteries due to intimal hyperplasia and medical hypertrophy; collateral venous-like channels were frequent (Fig. 1). No microemboli or thromboses were found. There was no evidence of vasculitis either in the lung or in the multiple sections of the left recurrent laryngeal nerve. There was right ventricular hypertrophy, but the cardiac valves were normal. The renal tubules contained proteinaceous casts, but there were no glomerular changes.

\section{Discussion}

Pulmonary hypertension in SLE without parenchymal lung disease is becoming increasingly well recognised. ${ }^{\text {T }}$ The mechanism for the development of pulmonary hypertension in such cases is not understood: the histological findings in the pulmonary vascular tree in our case were the same as those found in primary pulmonary hypertension. Vocal cord paralysis, when caused by pulmonary hypertension, is more commonly associated with mitral stenosis (Ortner's syndrome), and the anatomy of this has been described. ${ }^{2}$ Vocal cord paralysis in SLE or primary pulmonary hypertension is rare. The mechanism for the development of the recurrent laryngeal nerve palsy in this patient was direct compression of the nerve by the dilated pulmonary artery. A similar case was mentioned in a review of pulmonary hypertension in SLE, ${ }^{3}$ but it was thought that this might have been due to a mononeuritis secondary to vasculitis. Our patient did not have any evidence of vasculitis. The finding of more than two first degree relatives with SLE in a family is unusual. Contrary to the more usual finding of concordance of disease expression within families, ${ }^{4}$ our patient did not manifest any evidence of renal involvement, which had been the cause of death in her elder sister. Unfortunately tissue typing data are not available for the two sisters with the disease.

We thus report a case of rapidly progressive, fatal pulmonary hypertension complicated by recurrent laryngeal nerve palsy in a patient with SLE who had a strong family history of the disease.

\section{References}

1 Perez H. Kramer N. Pulmonary hypertension in SLE; report of four cases and a review of the literature. Semin Arthritis Rheum 1981: 11: 177-81

2 Morgan A A. Mourant A J. Left vocal cord paralysis and dysphagia in mitral valve disease. Br Heart $J$ 198(): 43: 470-3.

3 Asherson R A. Mackworth-Young C G. Bocy M L. et al. Pulmonary hypertension in SLE. Br Med J 1983; 287: 1024-5.

4 Arnett C F. Lawrence E S. Studies in familial SLE. Medicine (Baltimore) 1976: 55: 313-22. 